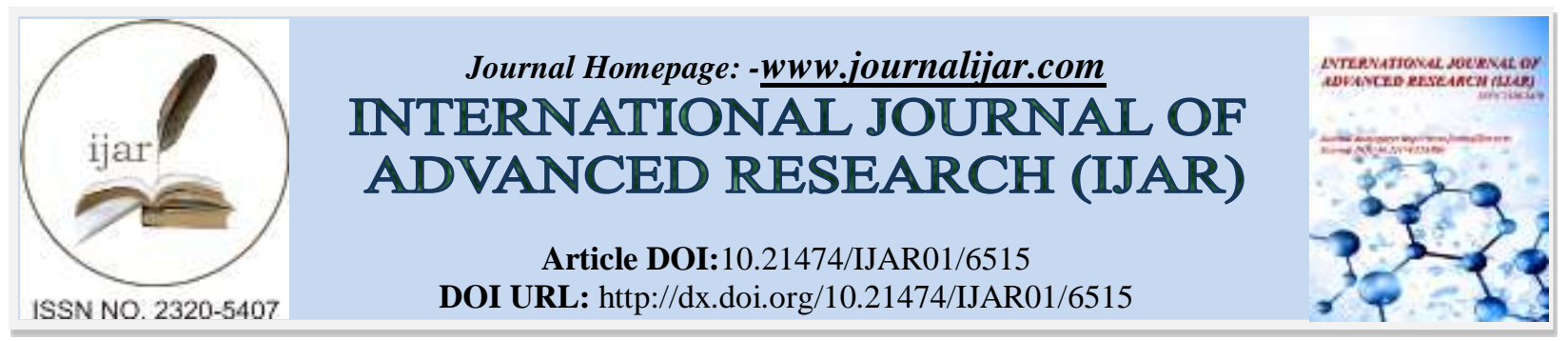

RESEARCH ARTICLE

\title{
ARTHRITIS AS MAIN PRESENTATION OF ACUTE LEUKAEMIA: A CASE REPORT.
}

\author{
Satyendra Kumar Sonkar ${ }^{1}$, Satish Kumar ${ }^{2}$ and Neeraj Kumar Singh ${ }^{3}$. \\ 1. Professor, Department Of Medicine, King George's Medical University, Lucknow, U.P., India. \\ 2. Senior Resident, Department Of Medicine, King George's Medical University, Lucknow, U.P., India. \\ 3. Junior Resident, Department Of Medicine, King George's Medical University, Lucknow, U.P., India.
}

\section{Manuscript Info}

..........................

Manuscript History

Received: 12 December 2017

Final Accepted: 14 January 2018

Published: February 2018

Keywords:-

Arthritis, Leukemia, Joint pain

\section{Abstract}

Background: Acute leukaemia presents with history of symptoms less than 6 weeks and joint involvement may be found in few cases. Joint involvement in leukaemia may be confused with transient synovitis, septic arthritis, osteomyelitis, reactive arthritis. It can create difficulty in the diagnosis of main disease since clinical, biochemical and radiological investigations are not pathognomic of leukaemia. It will cause delayed commencement of appropriate treatment and interventions and thus increase in severity of disease which will ultimately lead to mortality.

Case presentation: We report a case of a 22 year male who presented with a one and half month history of large joint polyarthritis which was a case of acute myeloid leukaemia masquerading as polyarthritis.

Copy Right, IJAR, 2018,. All rights reserved.

\section{Introduction:-}

Acute leukaemia presents with history of symptoms less than 6 weeks and joint involvement may be found in few cases. We report a case of a 22 year male who presented with a one and half month history of large joint polyarthritis. He had malaise, lethargy, mild grade fever and poor appetite but no history of night sweats. He was initially diagnosed as juvenile idiopathic arthritis, in which acute leukaemia became apparent after one and half month of presentation after being treated with steroids, NSAID and methotrexate. This case is important because this information will help clinician to look for cause of rather than implementing symptomatic treatment of joint pain.

\section{Case presentation:-}

A previously healthy 22 year old male patient was admitted with complaints of low grade fever, polyarthritis and generalised weakness since last one and half month. There was no history of diarrhoea, dysentery, dysuria and rash. On examination patient was afebrile and there was severe pallor but no lymphadenopathy or hepatosplenomegaly. Bilateral elbow, knee and ankle joints were tender and swollen and swelling was greater on right side. Previous blood investigations of one month back revealed haemoglobin of $11 \mathrm{gm} / \mathrm{dl}$, leukocytes $16400 \mathrm{cells} / \mathrm{mm}^{3}$, erythrocyte sedimentation rate (ESR) $08 \mathrm{~mm} / 1 \mathrm{hr}$. There was drastic change in blood investigations with clinical manifestations after one month which revealed haemoglobin of $5.2 \mathrm{gm} / \mathrm{dl}$, leukocytes 4900 cell $/ \mathrm{mm}^{3}$, ESR (Western Green) $119 \mathrm{~mm}$ in first hour, C- rective protein (CRP) $117 \mathrm{mg} / \mathrm{L}$ and viral markers were negative. Serum uric acid was $3.7 \mathrm{mg} / \mathrm{dl}$ (normal range 3.5-7.2 mg/dl). Antinuclear antibody, rheumatoid factor, Anti ds-DNA and serum complement level were within normal limits. The synovial fluid could not be aspirated during the first arthroscopy. The patient was prescribed indomethacin and colchicum for the pain, but during the following 2 weeks there was no benefit in the 
patient's symptoms. Pain and swelling of knees increased further with involvement of other peripheral joints. Arthroscopy was repeated and cytologic examination showed total cells 3500/cumm with polymorphs $50 \%$ and lymphocytes $24 \%$ without any blast cells or crystals. A bone marrow aspiration and biopsy was performed. Cells of granulocytic lineage were markedly increased and constitute $90 \%$ of all nucleated cells. 55\% of these were hyperchromatic cells with coarse chromatin, 1-2prominent nucleoid and scant amount of basophilic, granular cytoplasm. Auer rods were also seen occasionally. Cells of erythroid and megakaryocytic lineage were reduced in number. A diagnosis of acute myeloid leukaemia (AML) M2 was confirmed. Hence on investigations and clinical work up, diagnosis of acute leukaemia masquerading as polyarthritis was made.

\section{Differential diagnosis:-}

Joint involvement in leukaemia may be confused with transient synovitis, septic arthritis, osteomyelitis, reactive arthritis, juvenile rheumatoid arthritis (JRA). This may create diagnostic dilemma and delayed treatment.

\section{Treatment:-}

Initiation of appropriate chemotherapy resolves joint and bony symptoms but due to financial constraints treatment could not be instituted.

\section{Discussion:-}

Our case was a young adult male where at this age group, incidence of ALL and AML is nearly equal. ALL is most common in paediatric age group with peak age of occurrence between 2-6 years and it is more common in males while AML is evenly distributed in age and sex. ${ }^{[1]}$ Arthritis as main presentation of leukaemia is known from long time, and has been found to occur in $17 \%$ of new cases among chidren but few cases have been reported in young adults. ${ }^{[2]}$ Typical presentations are of asymmetrical large joint, oligoarticular arthritis which may be migratory in nature also. ${ }^{[2]}$ The presentation of leukaemia can mimic septic arthritis, reactive arthritis, osteomyelitis, transient synovitis, Lyme disease or juvenile rheumatoid arthritis and hence can cause difficulty in its diagnosis. ${ }^{[3]}$ In our patient, initial blood investigations did not showed anaemia, thrombocytopenia, leukopenia or blast cells in peripheral blood smear which would have been suggestive of leukemia. Hepatosplenomegaly, enlarged lymph nodes are found in $60 \%$ of cases but there was no such clinical finding in our case. Patient's joint symptoms did not improved and he became severely anaemic for which bone marrow aspiration was done to evaluate the cause of worsening pallor. Bone marrow examination revealed AML M2. In patients of AML in adults anemia, fever, haemorrhage or DIC are common presentation but our patient initially presented with arthritis which is very rare in young adults. ${ }^{[4]}$ Probably the mass proliferation of leukaemic cells is responsible for synovial effusion. It involves bone destruction, periostitis, juxtametaphysial demineralisation and effusions. Bony lytic lesions and diffuse infiltration causes osteopenia resembling osteomyelitis. In 47-69\% of cases these changes are visible at presentation and $70-90 \%$ during disease process. ${ }^{[5]}$

Joint symptoms are main presentation in children rather than bony pain, it may be monoarthritis or polyarthritis. Back symptoms can occur due to vertebral involvement and it may cause vertebral collapse which leads to fracture of vertebral column, the commonest cause of fracture in leukaemia. ${ }^{[6]}$ Joint swelling can also be due to haemorrhage because of thrombocytopenia. Immune complex induced synovitis may be another mechanism which may be attributable in our case. Initiation of appropriate chemotherapy resolves joint and bony symptoms but due to financial constraints treatment could not be instituted. ${ }^{[7]}$ Radiological changes are not pathognomonic. Clinical suspicion and haematological investigations are more valuable than radionucleide bone scans in making diagnosis. Small marrow of children is easily replaced by infiltrates and hence skeletal lesions are more frequent. In leukaemic arthritis patient, joint fluid aspiration can give early diagnosis of leukaemia by presence of blast cell but however it was not suggestive in our patient. Anaemia, leukaemia and non-diagnostic bone scan are the most helpful clues suggesting a possible diagnosis of leukaemia in these acute presentations. Bone marrow biopsy is diagnostic. Diagnosis is also confirmed by blast cells in peripheral blood smears but their absence does not exclude the diagnosis. ${ }^{[8]}$

\section{Learning points:-}

1. Clinician should look for cause of rather than to treat joint pain by pain killers.

2. Leukaemia must be excluded in juvenile arthritis before starting steroids or cytotoxic agents.

3. Clinician should keep differential diagnosis and investigations required to confirm or refute the diagnosis before commencement of treatment. 
4. The history and clinical examination can be misleading and haematological and radiological investigations are not pathognomonic at times.

5. The presence of anaemia, leukopenia, thrombocytopenia and a non-diagnostic bone scan are clues that should increase clinical suspicion of leukaemia as a cause for joint pathology.

6. Cytology examination of synovial fluid and bone marrow biopsy confirms the diagnosis of leukaemia.

\section{Footnotes:-}

Competing interest: none

Financial support: none

\section{References:-}

1. Wilson Anthony. Leukaemia in the young-Incidence and the survival statistics. Cancer news, Articles \& information- Health Hubs 2012.

2. Chell J, Fernandes JA, Bell MJ. The orthopaedic presentation of acute leukaemia in childhood. Ann R Coll Surg Engl 2001; 83: 186-89.

3. Usalan Celalettin, Ozarslan Ersan, Zengin Nurullah, Buyukayk Yahya, Gullu H Ybrahim. Acute lymphoblastic leukaemia presenting with arthritis in an adult patient. Postgraduate Medical Journal 2017;75:885.

4. Usalan C, Ozarslan E, Zengin N, Buyukayk Y, Gullu Y. Acute lymphoblastic leukaemia presenting with arthritis in an adult patient. Postgraduate Medical Journal. 1999;75(885):425-427.

5. D Sunita,Q Swee Tian, B Girish, J Pooja. Intra-articular and Peri-articular Tumours and Tumour Mimics- What a Clinician and Onco-imaging Radiologist Should Know. Malays J Med Sci. 2014 ; 21: 4-19.

6. Cabral DA, Tucker LB. Malignancies in children who initially present with rheumatic complaints. J Pediatr. 1999; 134(1):53-7.

7. Advani S Anjali, Hunger P Stephen, Burnett K Alan. Acute leukaemia in adolescents and young adults. Seminars in oncology 2009;36:213-26.

8. Murray M J, Tang T, Ryder C, Mabin D, Nicholsan JC. Childhood leukaemia masquerading as juvenile idiopathic arthritis.BMJ 2004;329:959-61. 